\title{
Flaring activity of Mrk 421 in 2012 and 2013: orphan flare and multiwavelength analysis
}

\author{
Nissim Fraija ${ }^{* \dagger}$ \\ Instituto de Astronomía, Universidad Nacional Autónoma de México \\ E-mail: nifraijaeastro.unam.mx \\ José Ignacio Cabrera \\ Facultad de Ciencias, Universidad Nacional Autónoma de México \\ E-mail: jcabreraeciencias. unam.mx
}

\section{Erika Benítez}

Instituto de Astronomía, Universidad Nacional Autónoma de México

E-mail: erika@astro.unam.mx

\section{David Hiriart}

Instituto de Astronomía, Universidad Nacional Autónoma de México

E-mail: hiriateastro.unam.mx

\begin{abstract}
The well-known blazar Mrk 421 is one of the most active and bright extragalactic sources in the Xray/ $\gamma$-ray sky. In 2012 and 2013, this object displayed two flares or high activity states detected by Fermi-LAT. The first one started in 2012 July 16 (MJD 56124) and the second one in 2013 April 9 (MJD 56391). The multiwavelength data analysis shows that the $\gamma$-ray flare observed in 2012 was not detected in the hard-X ray bands. This result is usually interpreted as an "orphan" flare. In 2013, the analysis of the multiwavelength light curves shows that there are two very bright states detected in the optical R-band. The first one in 2013 April $9(\mathrm{R}=11.74 \pm 0.04)$ and the second one in May $12(\mathrm{R}=11.62 \pm 0.04)$. Also, high activity states were detected in the soft and hard $\mathrm{X}$ rays. A discrete correlation function analysis of this last flare shows a strong correlation between the $\mathrm{GeV} \gamma$-rays and the optical/hard-X ray emission. These results are discussed in terms of the more adequate standard scenarios that could explain the multiwavelength variations displayed by this blazar.
\end{abstract}

Swift: 10 Years of Discovery,

2-5 December 2014

La Sapienza University, Rome, Italy

\footnotetext{
${ }^{*}$ Speaker.

${ }^{\dagger}$ Luc Binette postdoctoral scholarship.
} 


\section{Introduction}

At a distance of $134.1 \mathrm{Mpc}$, the BL Lac object Mrk $421(\mathrm{z}=0.03)$ [1] is one of the closest sources in the extragalactic sky. In the $\mathrm{MeV}-\mathrm{TeV}$ energy range, simultaneous observations have been carried out with the Fermi-LAT satellite ${ }^{1}$. This blazar was also studied using different telescopes based on Imaging Atmospheric Cherenkov Techniques (e.g. VERITAS, MAGIC, H.E.S.S.) and air shower arrays (e.g. ARGO-YBJ, HAWC). In X-rays, this object has been observed with Swift-XRT and Swift-BAT instruments for almost 10 years ${ }^{2}$. In the optical R-band, this source has been monitored since 2008 at San Pedro Mártir Observatory as part of the GASP-WEBT program ${ }^{3}$ [2]. On the other hand, VERITAS observatory and the Whipple $10 \mathrm{~m}$ Cherenkov telescope observed the flaring activity in $\mathrm{TeV} \gamma$-rays and studied the possible correlations with X-rays, optical and radio wavelengths between 2006 January and 2008 June [3]. Acciari et al. (2011) reported no significant flux correlations between the $\mathrm{TeV} \gamma$-rays and the optical/radio bands. However, an enhanced active phase in the X-ray and in $\mathrm{TeV} \gamma$-ray bands was observed. During this active phase, they found strong $\mathrm{X}$-ray activity with no increased $\mathrm{TeV}$ emission. Later, a $\mathrm{TeV} \gamma$-ray activity lasting two days was detected without activity in X-rays. Therefore, the later was associated with two "orphan" flares. At the end of this active state, the source showed a significant correlation between the X-ray and $\mathrm{TeV}$ bands. In general, no correlation in $\mathrm{TeV}$ has been found with optical and/or radio fluxes [3]. However, correlations among X-ray and $\mathrm{TeV}$ bands have been reported in [4, 5, 6]. Although most of these correlations are interpreted through the standard one-zone SSC model (synchrotron self-Compton; [7]), other correlations suggest serious deviations from this leptonic model.

The Spectral Energy Distribution (SED) of Mrk 421 presents a double-humped shape; a low energy hump at energies $\simeq 1 \mathrm{keV}$ and the second hump at hundreds of GeV. Abdo et al. (2011) found that both leptonic and hadronic scenarios are able to fit the Mrk 421 SED reasonably well, implying comparable jet powers but very different characteristics for the blazar emitting region. In the leptonic scenario, a one-zone SSC with three accelerated electron populations (through diffusive relativistic shocks with a randomly oriented magnetic field) has been used [8]. In the hadronic scenario [9], the peak at low energies is explained by electron synchrotron radiation whereas the high-energy peak is explained invoking the Synchrotron Proton Blazar (SPB) model [10, 11]. In this work, we show the multiwavelength observations carried out on Mrk 421 during the active states displayed in 2012 and 2013 in the GeV energy range. A brief discussion related to the theoretical interpretations of both flares will be given.

\section{Multiwalength Light Curves}

Multiwavelength light curves of the 2012 and 2013 flares of Mrk 421 are shown in Figure 1. The $\mathrm{GeV} \gamma$ - ray data shown corresponds to the $200 \mathrm{MeV}$ to $300 \mathrm{GeV}$ band. Details on the reduction procedure applied to these data-set can be found in [12]. The X-ray data were obtained with both Swift-BAT (15 - $50 \mathrm{keV})$ and Swift-XRT $(0.2-10 \mathrm{keV})$ instruments. The optical R-band observations were carried out with the $0.84 \mathrm{~m} \mathrm{f} / 15$ Ritchey-Chretien telescope and the instrument POLIMA

\footnotetext{
${ }^{1}$ http://fermi.gsf.nasa.gov/ssc/data/

${ }^{2}$ http://swift.gsfc.nasa.gov/cgi-bin/sdc/ql?

${ }^{3}$ http://www.oato.inaf.it/blazars/webt/
} 
${ }^{4}$. It is worth noting that the optical R-band magnitudes are not corrected for the contribution of the host galaxy of Mrk 421. Additionally, and only for comparison, we have included a few optical R-band data collected by the American Association of Variable Star Observers (AAVSO) ${ }^{5}$.

\subsection{Flare}

Mrk 421 was detected very active in the GeV energy range in July 16 with a daily flux of $(1.4 \pm 0.2) \times 10^{-6} \mathrm{ph} \mathrm{cm}^{-2} \mathrm{~s}^{-1}$, see ref. [13]. The source continued to be detected between July 17 and 21 , with a daily flux between $(0.4 \pm 0.1) \times 10^{-6} \mathrm{ph} \mathrm{cm}^{-2} \mathrm{~s}^{-1}$ and $(0.9 \pm 0.2) \times 10^{-6} \mathrm{ph} \mathrm{cm}^{-2}$ $\mathrm{s}^{-1}$. In fig. 1 we highlight the active state using a red vertical bar. It is clear that $\gamma$-ray flare was detected without activity in the hard X-ray band. Unfortunately, data were collected neither in the soft-X rays nor the optical R-band.

\subsection{Flare}

Fermi-LAT reported high activity of Mrk 421 in 2013 April 9 to 12, with a daily flux between $(0.4 \pm 0.1) \times 10^{-6} \mathrm{ph} \mathrm{cm}^{-2} \mathrm{~s}^{-1}$ and $(0.8 \pm 0.2) \times 10^{-6} \mathrm{ph} \mathrm{cm}^{-2} \mathrm{~s}^{-1}$, see ref. [14]. In fig. 1, the states of high activity have been divided in two vertical color bands, purple and green. The purple one marks the high activity observed in GeV $\gamma$-rays, X-rays (XRT and BAT) and optical bright R-band (April 9, $\mathrm{R}=11.74 \pm 0.04$ ). The green color vertical band marks the second bright optical R-band point (May 12, $R=11.62 \pm 0.04$ ). The second optical bright point seems to be anti-correlated with the higher energy bands.

\section{Discussion}

From the multiwavelength light curves it is clear that the $\gamma$-ray flare observed in 2012 was detected without any strong activity in the hard-X rays. In 2012 July 16, a TeV $\gamma$-ray flare without any increased activity in other wavelengths was reported [15]. The so-called "orphan" flares have been previously observed in Mrk 421 [16, 3], and also in the blazar 1ES 1959+650 [17, 18]. In general, most of the flaring activity in this source occurs quasi-simultaneously with $\gamma$-ray and $\mathrm{X}$-ray emission. Therefore, this atypical flaring event observed in the $\mathrm{TeV} / \mathrm{GeV} \gamma$-rays along with the absence of activity in the X-rays is very difficult to reconcile with the SSC model. Orphan flares have been usually explained as due to neutral pion decays from proton-photon interactions $[19,20,21]$. It is worth mentioning that a radio flare with a delay of $\sim 60$ days was detected by the Owens Valley Radio Observatory (OVRO) 40-m Telescope [22, 23].

Based on the multiwavelength light curves shown in fig. 1, and the emission in $\mathrm{TeV}$ and $\mathrm{X}$ ray reported by $[24,25]$, it is clear that Mrk 421 flared in $\mathrm{TeV} / \mathrm{GeV} \gamma$-rays, in X-rays (BAT and XRT) and in optical R-band in 2013 April 9 - 12. The results obtained from the discrete correlation function (DCF) calculated using all the light curves in this work are presented in Figure 2. Left panel shows the correlation between $\mathrm{GeV} \gamma$-rays and hard-X rays, and the right panel shows the

\footnotetext{
${ }^{4} \mathrm{~A}$ detailed description of our photopolarimetric monitoring program on $\mathrm{TeV}$ blazars can be found in http://www.astrossp.unam.mx/blazars

${ }^{5}$ http://www.aavso.org/observing-campaigns
} 
correlation between $\mathrm{GeV} \gamma$-rays and the optical R-band. In both panels the DCF show that there are no lags between the $\mathrm{GeV} \gamma$-rays and hard-X rays, and also with the optical R-band. Therefore, the multiwavelength emission seems to take place simultaneously in all bands, which favors a one-zone SSC model. In the framework of this SSC model, the electrons within the emitting region are moving at ultra-relativistic velocities in a collimated jet. The Fermi-accelerated electrons injected into the emitting region are confined by a magnetic field. Then, photons are radiated via synchrotron emission and up-scattered to higher energies. The low energy emission, from radio to $\mathrm{X}$-rays, is produced by synchrotron radiation. The high energy emission $(\mathrm{MeV}-\mathrm{TeV}) \gamma$-rays, is due to Compton scattering. This leptonic model depends basically on the bulk Lorentz factor, the size of emitting region, the electron number density and the strength of the magnetic field. It is possible to find a set of parameters that can describe the states of low or high activity [3].

It is worth noting that the maximum brightness in the optical R-band observed in May 12 is anti-correlated with the other bands. This result poses a challenge for the theoretical models proposed for this blazar. In a forthcoming paper, we will present a more detailed analysis of these active states.

\section{References}

[1] B. Sbarufatti, A. Treves and R. Falomo, Imaging Redshifts of BL Lacertae Objects, ApJ 635 (2005) 173

[2] M. Villata et al., Multifrequency monitoring of the blazar 0716+714 during the GASP-WEBT-AGILE campaign of 2007, A\&A 481 (2008) L79

[3] V. A. Acciari et al., TeV and Multi-wavelength observations of Mrk 421 in 2006 - 2008, ApJ 738 (2011) 25

[4] D. Macomb, C. Akerlof and H. D. Aller, Multiwavelength Observations of Markarian 421 During a TeV/X-Ray Flare, ApJ 449 (1995) L99

[5] J. Kildea and et al., Multiwavelength observations of Markarian 421 in 2001 March: An unprecedented view on the X-ray/TeV correlated variability, ApJ 677 (2008) 906

[6] D. Horan, V. A. Acciari and S. M. Bradbury, Multiwavelength Observations of Markarian 421 in 2005-2006, ApJ 695 (2009) 596

[7] J. D. Finke, C. D. Dermer, and M. Böttcher, Synchrotron Self-Compton Analysis of TeV $X$-Ray-Selected BL Lacertae Objects, ApJ 686 (2008) 181

[8] A. A. Abdo and et al., Fermi Large Area Telescope Observations of Markarian 421: The Missing Piece of its Spectral Energy Distribution, ApJ 736 (2011) 131

[9] N. Fraija and A. Marinelli, TeV $\gamma$-ray fluxes from the long campaigns on Mrk421 as constraints on the emission of TeV-PeV Neutrinos and UHECRs, Astroparticle Physics 70 (2015) 54

[10] A. Mücke and R. J. Protheroe, A proton synchrotron blazar model for flaring in Markarian 501, Astroparticle Physics 15 (2001) 121

[11] A. Mücke and et al., BL Lac objects in the synchrotron proton blazar model, Astroparticle Physics $\mathbf{1 8}$ (2003) 593 


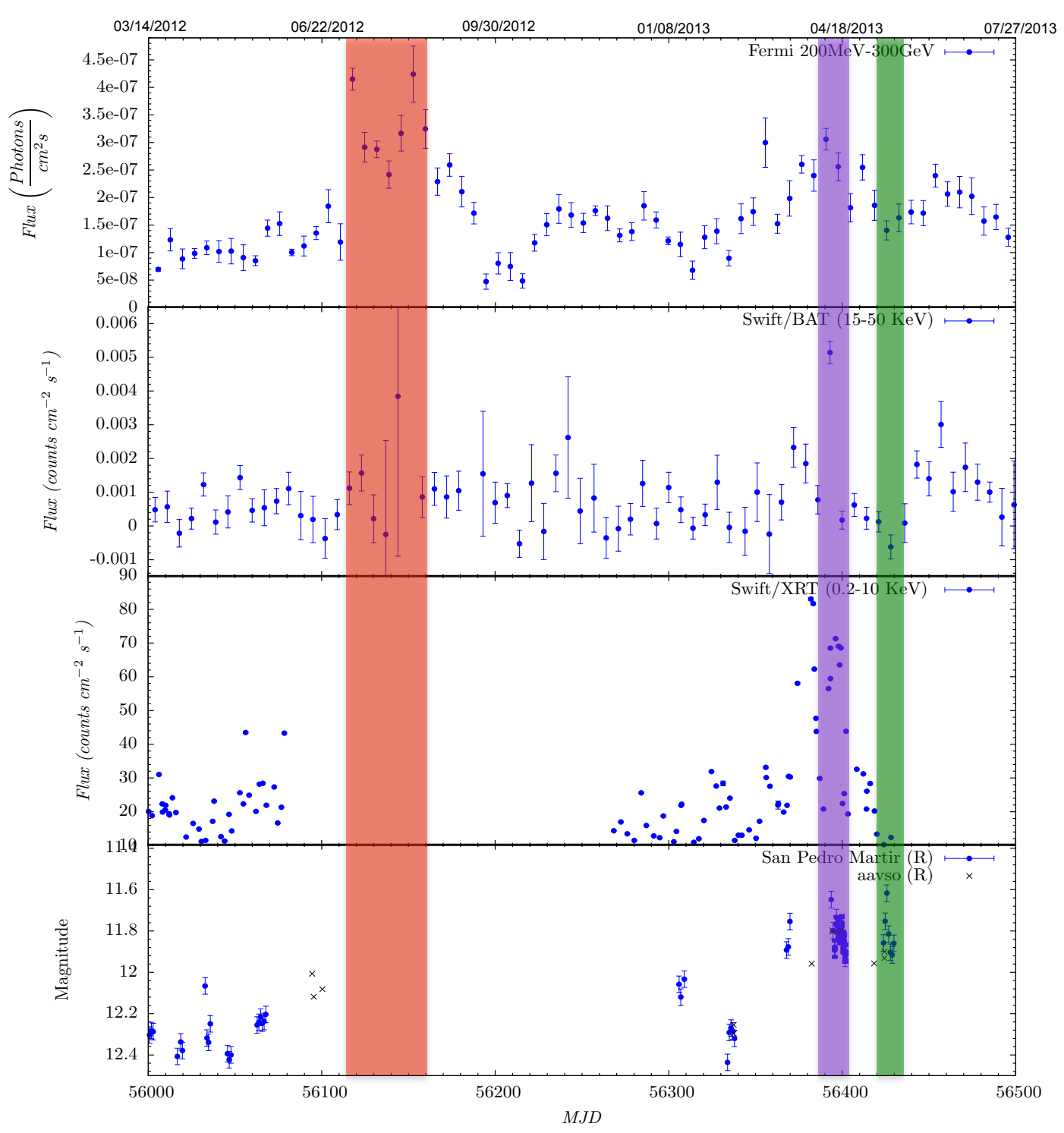

Figure 1: Mrk 421 light curves obtained with Fermi, Swift and San Pedro Mártir data in 2012-2013. From top to bottom: GeV $\gamma$-rays, hard-X rays, soft-X rays and the optical R-band.

[12] J. I. Cabrera et al., A hydrodynamical model for the Fermi-LAT $\gamma$-ray light curve of blazar PKS 1510-089, MNRAS, 434 (2013) L6

[13] F. D'Ammando and M. Orienti, Fermi LAT detection of a GeV flare from the BL Lac object Mrk 421, The Astronomer's Telegram 4261 (2012) 1

[14] D. Paneque et al., Fermi-LAT and Swift-XRT observe exceptionally high activity from the nearby TeV blazar Mrk421, The Astronomer's Telegram 4977 (2013) 1

[15] B. Bartoli and et al., TeV flare from the blazar Mrk421 observed by ARGO-YBJ, The Astronomer's Telegram, 4272 (2012) 1

[16] M. B łażejowski et al., A Multiwavelength View of the TeV Blazar Markarian 421: Correlated Variability, Flaring, and Spectral Evolution, ApJ 630 (2005) 130 

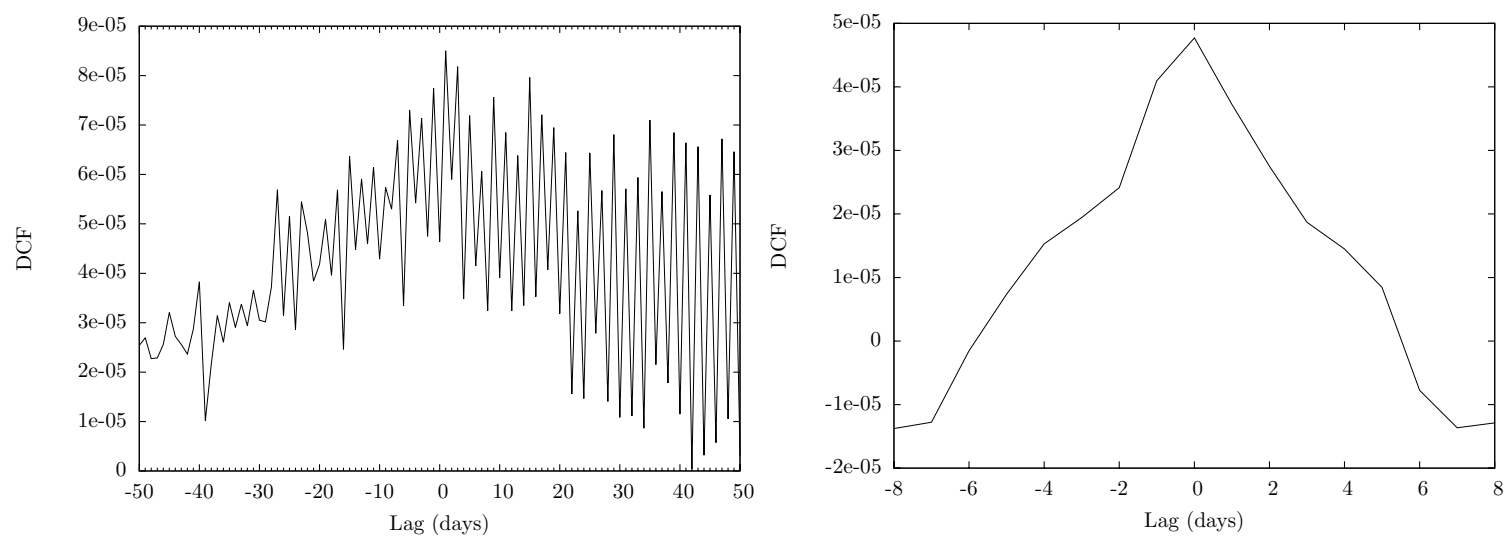

Figure 2: Left: DCF between the GeV $\gamma$-rays and X-rays. Right: DCF between the GeV $\gamma$-rays and the optical R-Band.

[17] J. Holder and et al., Detection of TeV Gamma Rays from the BL Lacertae Object 1ES 1959+650 with the Whipple 10 Meter Telescope, ApJ 583 (2003) L9

[18] H. Krawczynski and et al., Multiwavelength Observations of Strong Flares from the TeV Blazar 1ES $1959+650$, ApJ 601 (2004) 151

[19] M. Böttcher, A Hadronic Synchrotron Mirror Model for the “Orphan” TeV Flare in 1ES 1959+650, ApJ 621 (2005) 176

[20] S. Sahu, A. F. Oliveros and J. C. Sanabria, Hadronic-origin orphan TeV flare from 1ES 1959+650, PRD 87 (2013) 10

[21] N. Fraija, Could a plasma in quasi-thermal equilibrium be associated to the "orphan" TeV flares?, Astroparticle Physics 71 (2015) 1

[22] T. Hovatta et al., A major 15 GHz radio flare in the blazar Mrk 421, The Astronomer's Telegram 4451 (2012) 1

[23] T. Hovatta et al., A combined radio and GeV gamma-ray view of the 2012 and 2013 flares of Mrk 421, [hep-th/1501.07407]

[24] J. Cortina and J. Holder, MAGIC and VERITAS detect an unprecedented flaring activity from Mrk 421 in very high energy gamma-rays, The Astronomer's Telegram 4976 (2013) 1

[25] E. Pian et al., An active state of the BL Lacertae object Markarian 421 detected by INTEGRAL in April 2013, A\&A $\mathbf{5 7 0}$ (2014) 77 